\title{
Targeting mutant KRAS with CRISPR-Cas9 controls tumor growth
}

\author{
Wonjoo Kim, ${ }^{1}$ Sangeun Lee, ${ }^{1,2}$ Han Sang Kim, ${ }^{1,2,3}$ Minjung Song, ${ }^{1,4}$ \\ Yong Hoon Cha, ${ }^{5,6}$ Young-Hoon Kim, ${ }^{1,7,8}$ Jeonghong Shin, ${ }^{1,2,9,10}$ Eun-Seo Lee, ${ }^{1,11}$ \\ Yeonsoo Joo, ${ }^{12}$ Jae J. Song, ${ }^{12}$ Eun Ju Choi, ${ }^{13}$ Jae W. Choi, ${ }^{12}$ jinu Lee, ${ }^{13}$ \\ Moonkyung Kang, ${ }^{14}$ Jong In Yook, ${ }^{5}$ Min Goo Lee, ${ }^{1,2}$ Yeon-Soo Kim, ${ }^{14}$ \\ Soonmyung Paik, ${ }^{12}$ and Hyongbum (Henry) Kim ${ }^{1,2,7,9,10,12}$
}

${ }^{1}$ Department of Pharmacology, Yonsei University College of Medicine, Seoul, 03722, South Korea; ${ }^{2}$ Brain Korea 21 Plus Project for Medical Sciences, Yonsei University College of Medicine, Seoul, 03722, South Korea; ${ }^{3}$ Division of Medical Oncology, Department of Internal Medicine, Yonsei University College of Medicine, Seoul, 03722, South Korea; ${ }^{4}$ Department of Food Biotechnology, Division of Bioindustry, College of Medical and Life Sciences, Silla University, Busan, 46958, South Korea; ${ }^{5}$ Department of Oral Pathology, Oral Cancer Research Institute, College of Dentistry, Yonsei University, Seoul, 03722, South Korea; ${ }^{6}$ Department of Oral and Maxillofacial Surgery, College of Dentistry, Yonsei University, Seoul, 03722, South Korea; ${ }^{7}$ Graduate Program of Nano Science and Technology, Yonsei University, Seoul, 03722, South Korea; ${ }^{8}$ ToolGen, Seoul, 08501, South Korea; ${ }^{9}$ Center for Nanomedicine, Institute for Basic Science (IBS), Seoul, 03722, Republic of Korea; ${ }^{10}$ Yonsei-IBS Institute, Yonsei University, Seoul, 03722, Republic of Korea; ${ }^{11}$ School of Chemical and Biological Engineering, Seoul National University, Seoul, 08826, South Korea; ${ }^{12}$ Severance Biomedical Science Institute, Yonsei University College of Medicine, Seoul, 03722, South Korea; ${ }^{13}$ College of Pharmacy, Yonsei Institute of Pharmaceutical Sciences, Yonsei University, Incheon, 21983, South Korea; ${ }^{14}$ Graduate School of New Drug Discovery \& Development, Chungnam National University, Daejeon 305-763, South Korea

KRAS is the most frequently mutated oncogene in human tumors, and its activating mutations represent important therapeutic targets. The combination of Cas9 and guide RNA from the CRISPR-Cas system recognizes a specific DNA sequence and makes a double-strand break, which enables editing of the relevant genes. Here, we harnessed CRISPR to specifically target mutant KRAS alleles in cancer cells. We screened guide RNAs using a reporter system and validated them in cancer cells after lentiviral delivery of Cas9 and guide RNA. The survival, proliferation, and tumorigenicity of cancer cells in vitro and the growth of tumors in vivo were determined after delivery of Cas9 and guide RNA. We identified guide RNAs that efficiently target mutant KRAS without significant alterations of the wild-type allele. Doxycycline-inducible expression of this guide RNA in KRAS-mutant cancer cells transduced with a lentiviral vector encoding Cas9 disrupted the mutant KRAS gene, leading to inhibition of cancer cell proliferation both in vitro and in vivo. Intra-tumoral injection of lentivirus and adenoassociated virus expressing Cas9 and sgRNA suppressed tumor growth in vivo, albeit incompletely, in immunodeficient mice. Expression of Cas9 and the guide RNA in cells containing wild-type KRAS did not alter cell survival or proliferation either in vitro and in vivo. Our study provides a proof-of-concept that CRISPR can be utilized to target driver mutations of cancers in vitro and in vivo.

[Supplemental material is available for this article.]

The RAS subfamily member KRAS is the most frequently mutated oncogene in cancers, including highly lethal lung, colon, and pancreatic cancers (Cox et al. 2014). Activating mutations in KRAS play potent roles in cancer initiation, propagation, and maintenance, representing important therapeutic targets (Cox et al. 2014). However, despite more than three decades of substantial effort by academia and industry, activating $K R A S$ mutations remain a critical challenge because few effective and specific inhibitors for these mutations are available, necessitating other approaches to target KRAS mutations. Although the obstacle of targeting mutant $K R A S$ could be partly overcome with the recent development of $\mathrm{KRAS}^{\mathrm{G} 12 \mathrm{C}}$-specific inhibitors (Ostrem et al. 2013; Patricelli et al. 2016) and a RAS-binding domain inhibitor that is not selective

Corresponding author: hkim1@yuhs.ac

Article published online before print. Article, supplemental material, and publication date are at http://www.genome.org/cgi/doi/10.1101/gr.223891.117. for mutant RAS (Athuluri-Divakar et al. 2016), mutation-specific inhibition was not achieved using small molecules for KRAS ${ }^{\mathrm{G} 12 \mathrm{D}}$ or $\mathrm{KRAS}^{\mathrm{G} 12 \mathrm{~V}}$, which occur more frequently than $\mathrm{KRAS}^{\mathrm{G} 12 \mathrm{C}}$. Small interfering RNAs (siRNAs) that selectively inhibit mutant KRAS mRNAs (Brummelkamp et al. 2002), but not their origin DNA, lead to knockdown, rather than complete knockout, of the mutant mRNA, and their continuous expression or delivery is required for target RNA suppression (Zorde Khvalevsky et al. 2013). Methods for targeting the oncogenic KRAS mutations themselves in cancer cells in vivo have yet to be reported.

Programmable nucleases, which include zinc finger nucleases, transcriptional activator-like effector nucleases, and CRISPR-

C $2018 \mathrm{Kim}$ et al. This article is distributed exclusively by Cold Spring Harbor Laboratory Press for the first six months after the full-issue publication date (see http://genome.cshlp.org/site/misc/terms.xhtml). After six months, it is available under a Creative Commons License (Attribution-NonCommercial 4.0 International), as described at http://creativecommons.org/licenses/by-nc/4.0/. 
Cas (clustered regularly interspaced short palindromic repeats and CRISPR-associated proteins), recognize specific DNA sequences and make double-strand breaks, the repair of which can lead to indel formation at the target sites by error-prone nonhomologous end joining (NHEJ) (Jinek et al. 2012; Cong et al. 2013; Mali et al. 2013; Kim and Kim 2014). This indel formation can lead to disruption of the corresponding gene (Jinek et al. 2012; Cong et al. 2013; Mali et al. 2013; Kim and Kim 2014; Kim et al. 2015). Thus, we expected that we could disrupt mutant KRAS alleles, but not wild-type KRAS, using Cas9 and guide RNAs that specifically recognize the mutant sequences.

Here, we attempted to identify guide RNAs that specifically target mutant, but not wild-type, KRAS. We also evaluated whether the delivery of Cas 9 and a guide RNA targeting mutant KRAS into cancer cells would affect the survival, proliferation, and tumorigenicity of the cultured cancer cells in vitro and the tumor growth in vivo.

\section{Results}

\section{Selection of guide RNAs that target mutant KRAS}

To target mutant KRAS alleles in cancer cells with the CRISPR-Cas9 system, we first screened for guide RNAs that specifically target $K R A S$ mutations, including c.35G $>$ T (p.G12V), c.35G $>$ A (p.G12D), and c.38G $>$ A (p.G13D) (Supplemental Fig. S1), but not wild-type KRAS. Most cancer cell lines containing KRAS mutations also have a wild-type KRAS allele in their genome; indels generated at the mutant allele are hard to distinguish from indels at the wild-type allele, making it difficult to find mutant-specific guide RNAs using these cell lines. Thus, we adopted our surrogate NHEJ reporter system that enables the evaluation of guide RNA activity (Kim et al. 2011; Ramakrishna et al. 2014). These reporters contain a monomeric red fluorescent protein ( $m R F P$ ) gene, CRISPR-Cas9 target sequences, and two enhanced green fluorescent protein $(e G F P)$ genes (Supplemental Fig. S2). The reporters express eGFP only when frame-shifting indels such as $(3 n+1)$ or $(3 n+2)$ nucleotide insertions or deletions are generated at the target sequences in the reporter plasmid. To identify guide RNAs that specifically and efficiently target mutant, but not wild-type, KRAS, we cotransfected a reporter plasmid containing the mutant or wild-type $K R A S$ sequence as the target, a plasmid encoding Cas9, and a plasmid encoding a single guide RNA (sgRNA) into HEK293T cells. We then subjected the transfected cells to flow cytometry shown in bold. to determine the percentage of cells expressing both mRFP and eGFP normalized to that of cells only expressing mRFP, which represents the activity of the guide RNA on the target sequence. Some guide RNAs resulted in high GFP expression with the mutant target sequence and low expression with the wild-type sequence (Fig. 1; Supplemental Fig. S3), suggesting that they were selectively and efficiently targeting the mutant KRAS sequences. For subsequent studies, we selected three guide RNAs: two with high targeting selectivity (35T9P17 and 38A6P17) and one with low targeting selectivity (35A9P17) for mutant KRAS.
A

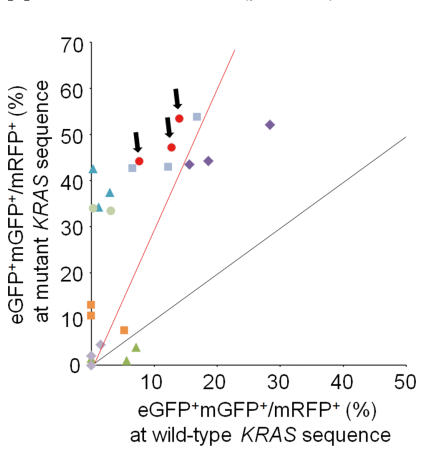

B

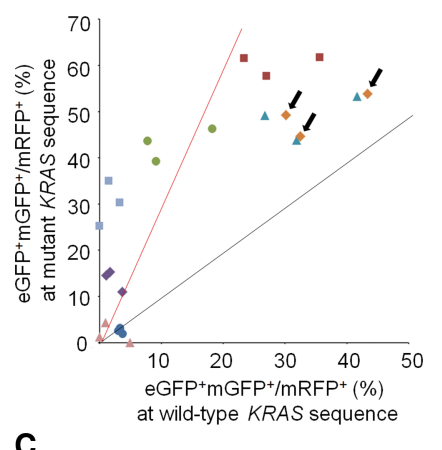

C

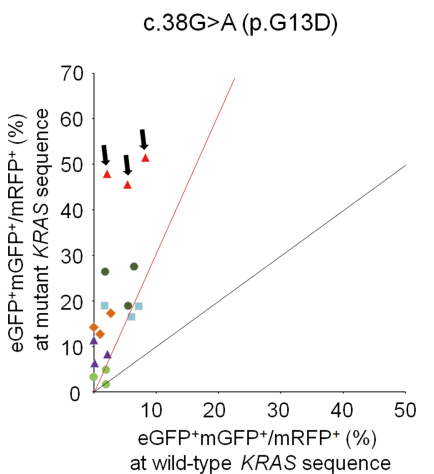

Wild-type KRAS CTTGTGGTAGTTGGAGCTGGTGGCGTAGGCAAGAGTGCC c.38G>A Mutant CTTGTGGTAGTTGGAGCTGGTGACGTAGGCAAGAGTGCC

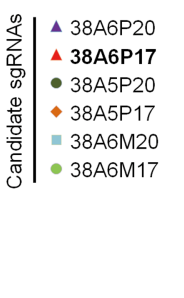

Figure 1. Reporter-based selection of sgRNAs that target mutant KRAS. The ratios of eGFP+mRFP $+/$ eGFP+ cells after transfection of reporters with mutant (c.35G >T [A], c.35G>A [B], or c.38G>A $[C]$ ) or wild-type target sequences, which reflect guide RNA activities at the target sequences, are shown on the left in the two-dimensional graphs. Black and red lines in the graphs represent ratios of eGFP+mRFP+/mRFP+ cells for mutant vs. wild-type KRAS target sequences with values of 1 and 3 , respectively. The target sequence of each sgRNA is shown on the right side; the KRAS point mutation site is shown in red and the protospacer adjacent motif sequence is in blue. sgRNAs selected for subsequent studies are indicated with black arrows in the two-dimensional graphs and their names are 


\section{Cas9 and sgRNA-directed selective and efficient disruption of mutant KRAS in cancer cells}

To validate the function of these selected guide RNAs at the endogenous targets, we next transduced cancer cells containing these mutations with lentiviral vectors encoding Cas9 and the corresponding guide RNAs (Supplemental Fig. S4). Deep sequencing showed that delivery of Cas9 and the 35T9P17 sgRNA resulted in indel frequencies of 50\% in SW403 cells, which are heterozygous for the c.35G>T mutation, and $81 \%$ and $80 \%$ in SW 480 and SW620 cells, respectively, which are homozygous for the c.35G $>\mathrm{T}$ mutation, leading to a drastic reduction of mutant KRAS: from $70 \%, 100 \%, 100 \%$ to $12 \%, 19 \%, 20 \%$, respectively (Fig. 2A-C, G; Supplemental Figs. S5A-C, S6A). Similarly, lentiviral delivery of Cas9 and 35A9P17 or 38A6P17 into LS513 cells, which are heterozygous for the c.35G $>$ A mutation, or LoVo cells, which are heterozygous for the c.38G $>$ A mutation, leads to indel frequencies of $36 \%$ or $28 \%$, respectively (Fig. 2D,E; Supplemental Fig. $\mathrm{S} 5 \mathrm{D}, \mathrm{E})$. As a result, mutant KRAS was reduced from $53 \%$ or $67 \%$ to $20 \%$ or $22 \%$ (Fig. 2G; Supplemental Fig. S6B,C). When these guide RNAs were lentivirally delivered into HT29 cells, which contain wild-type KRAS, the indel frequencies were $0.2 \%$ for 35T9P17, 77\% for 35A9P17, and 0.3\% for 38A6P17 (Fig. 2F; Supplemental Fig. S5F), indicating that 35T9P17 and 38A6P17, but not 35A9P17, are highly selective for mutant, but not wildtype, KRAS. These indel frequencies at the endogenous sites are basically compatible with the reporter assay results described above. When we conducted a similar test using a lentivirus that expresses 35T9P17 in a doxycycline-inducible manner, we observed similar results (Supplemental Fig. S7), corroborating that 35T9P17 is a highly selective and efficient guide RNA targeting c.35G $>\mathrm{T}$ KRAS (p.G12V).
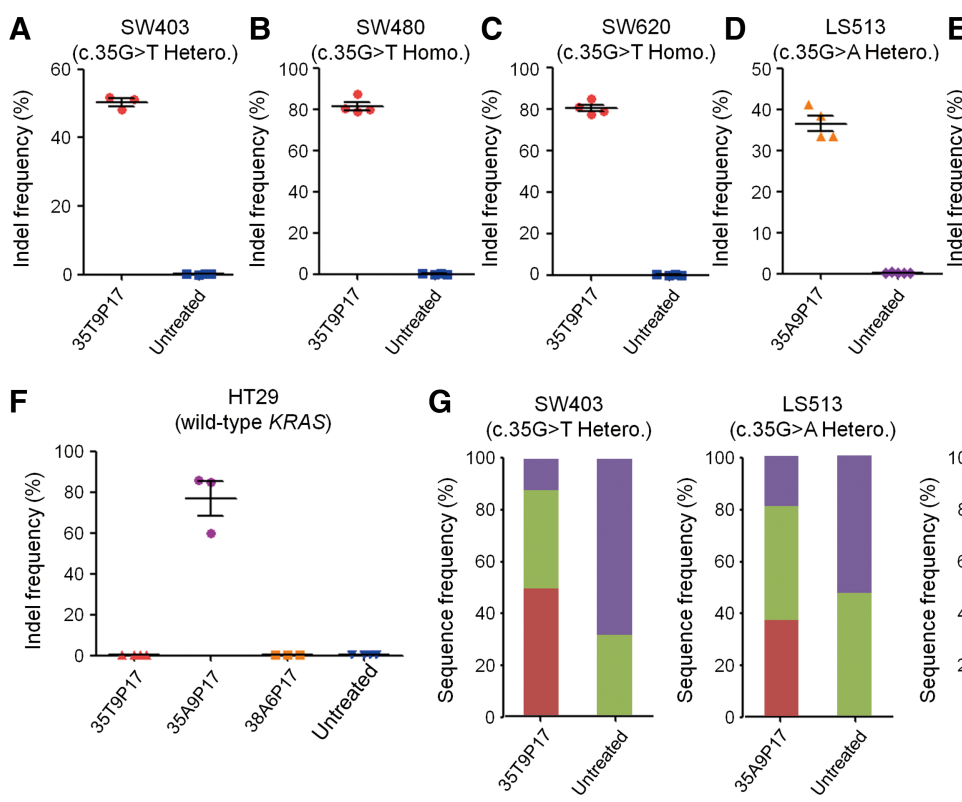

Figure 2. Cas 9 and sgRNA-directed selective and efficient disruption of mutant KRAS in cancer cells. Cas9 and sgRNAs were delivered into cancer cells and the indel frequencies at the endogenous target sequences were evaluated by deep sequencing. Untreated cells were used as the control. The names of the cancer cell lines and their related mutations are shown at the top of each graph. (Hetero.) Heterozygous, (Homo.) homozygous. (A-F) Indel frequencies in cancer cells after delivery of Cas9 and sgRNA. Error bars represent SEM. (G) Average sequence frequencies. The values from each experiment are shown in Supplemental Figure S6. Red = Indels, green = wild-type KRAS (unedited), purple = mutant KRAS (unedited).
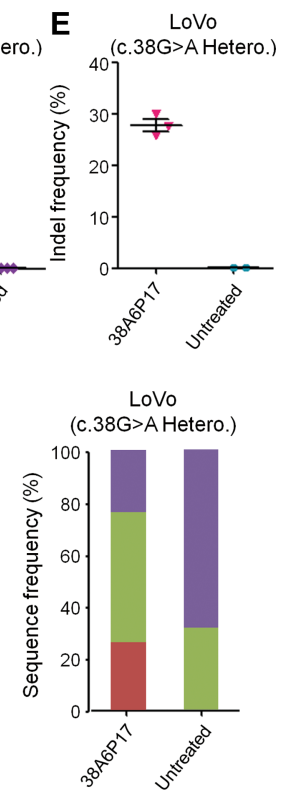

When we analyzed the indel sequences, we identified a diverse set of sequences and observed that roughly one third of them were in-frame (Supplemental Fig. S8). Given that the senear the cleavage site encode an important functional domain of KRAS (Ostrem et al. 2013; Hunter et al. 2015) and that targeting functional domains can lead to loss-of-function even when in-frame variants are generated (Shi et al. 2015), these

\section{Targeting mutant KRAS with CRISPR-Cas9 inhibits cancer} cell survival, proliferation, and tumorigenicity in vitro

e next determined whether such disruption of mutant KRAS in vitro. Cancer cells were first transduced with Cas9-encoding lentiviral vectors and then transduced with guide RNA-encoding vecsoft agar assays. Expression of Cas9 and the 35T9P17 sgRNA in SW403 cells, which are heterozygous for c.35G>T, led to $94 \%$ and soft agar assays, respectively, suggesting that disruption of mutant KRAS using Cas9 and sgRNA inhibits the survival and tumorFig. S9A-D). Furthermore, expression of Cas9 and the 35A9P17 sgRNA in LS513 cells, which are heterozygous for c.35G $>$ A, resulted in 91\% and 96\% reductions in the number of colonies in colony forming and soft agar assays, respectively, indicating inhibition of survival and tumorigenicity (Fig. 3B,F). The expression of Cas9 and the 38A6P17 sgRNA also reduced the number of colonies in both assays, but the reduction was, albeit significant, only $25 \%$ and $61 \%$, respectively (Fig. 3C,G), suggesting that a sgRNA with low activity only partially inhibits the survival and tumorigenicity of KRAS mutant cancer cells. The expression of Cas9 and these sgRNAs in HT29 cells did not change the number of colonies in either assay (Fig. 3D,H), suggesting that reduction of survival and tumorigenicity by Cas9 and these sgRNA is restricted to KRAS mutant cells. In addition, we expressed guide RNA in a doxycycline-inducible manner in cells transduced with a lentiviral vector that constitutively expresses Cas9. This doxycycline-inducible guide RNA expression selectively inhibited colony formation in SW403, SW480, and SW620 cells, which contain c.35G $>$ T KRAS, but not in HT29 cells, which contain wild-type KRAS (Supplemental Fig. S10), corroborating that Cas9 and the 35T9P17 sgRNA selectively suppress survival and tumorigenicity of KRAS c.35G > T mutant cells, but not cells containing wild-type $K R A S$, in vitro.

We also evaluated the effect of Cas9 and guide RNA on cell proliferation using MTS and xCELLigence cell proliferation

\section{Genome Research}

www.genome.org 
assays. After lentiviral delivery of Cas9 and guide RNA, live cells were counted and 5000 cells were plated into 96-well (for the MTS assay) or 16-well plates (for the xCELLigence assay). Performance of the MTS assay at $48 \mathrm{~h}$ after plating showed that the relative viable cell number in the population expressing Cas9 and the 35T9P17 RNA were, on average, $0.34,0.46$, and 0.71 in SW403, SW480, and SW620 cells, respectively (Fig. 3I; Supplemen- tal Fig. S9E,F), suggesting that mutant KRAS disruption using Cas9 and sgNA inhibits cell proliferation or survival. Similarly, lentiviral delivery of Cas9 and 35A9P17 sgRNA into LS513 cells and that of Cas9 and 38A6P17 sgRNA into LoVo cells resulted in a significant reduction of the relative viable cell number to 0.18 and 0.69 , respectively (Fig. 3J,K). A real-time cell proliferation assay using xCELLigence showed that the cell index started to decrease about
A

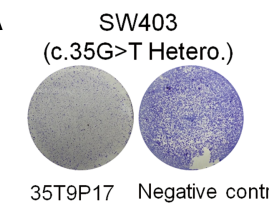

$\begin{array}{r}\text { 35T9P17 Negative } \\ * * * \\ \hline\end{array}$

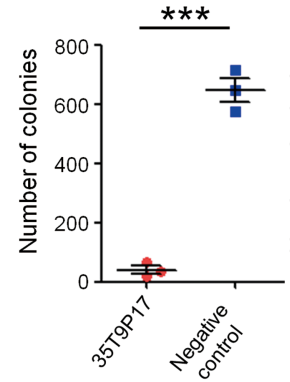

E

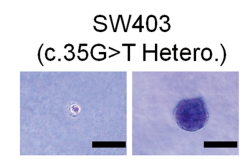

35T9P17 Negative control
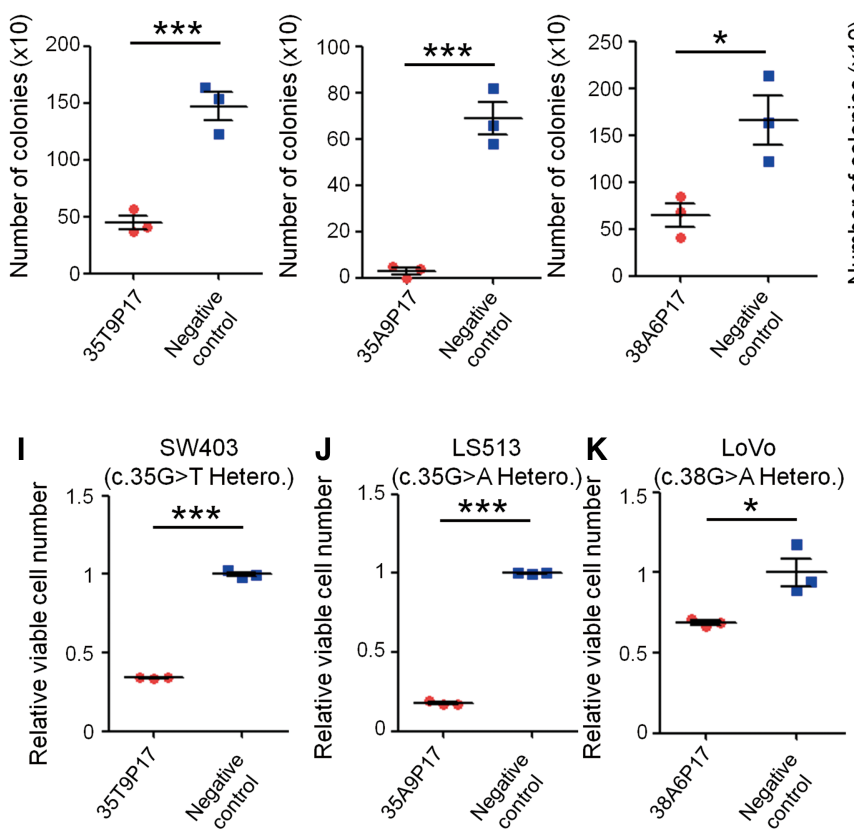

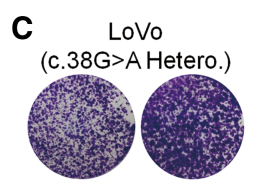

35A9P17 Negative control 38A6P17 Negative contro
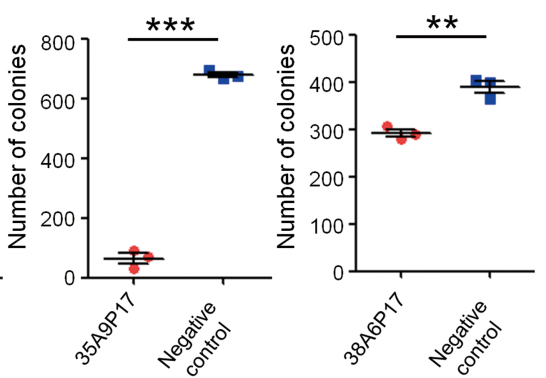

F LS513

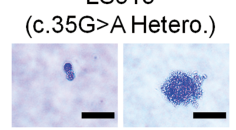

G

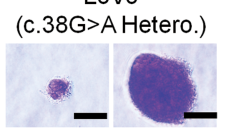

38A6P17 Negative control

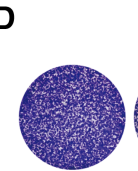

HT29

(wild-type KRAS)

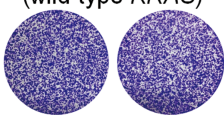

35A9P17

38A6P17

egative

control
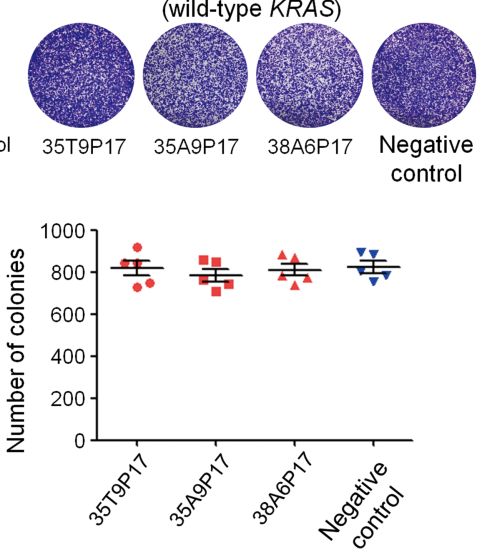

H

HT29

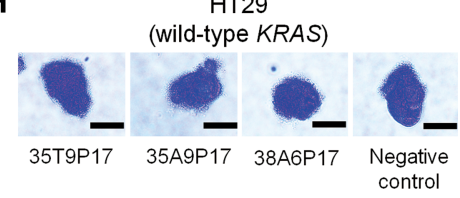

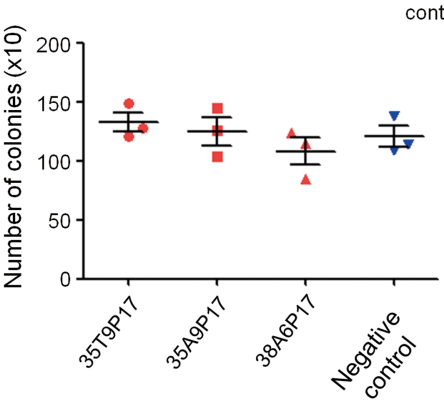

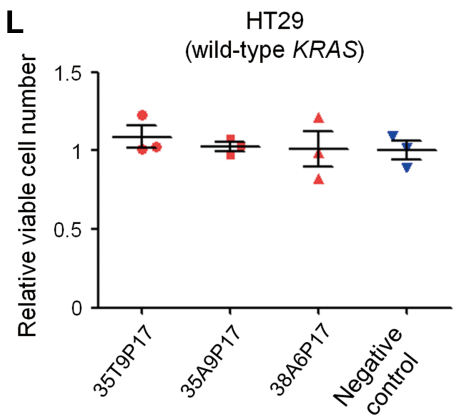

Figure 3. Targeting mutant KRAS with CRISPR-Cas9 inhibits cancer cell survival, proliferation, and tumorigenicity in vitro. Cancer cells containing KRAS mutations were subjected to colony forming $(A-D)$, soft agar $(E-H)$, and MTS $(I-L)$ assays after lentiviral delivery of Cas 9 and sgRNAs targeting mutant $K R A S$. A completely different sequence-targeting guide RNA without activity was used as the control (negative control). (Hetero.) Heterozygous, (Homo.) homozygous. Error bars represent SEM. $\left({ }^{*}\right) P<0.05,\left(^{* *}\right) P<0.01,\left({ }^{* *}\right) P<0.001$. (A-D) Colony forming assay. Representative images of wells after $2 \%$ crystal violet staining are shown at the top of each graph. $(E-H)$ Soft agar assay. Representative images of formed colonies are shown at the top of each graph. Scale bar $=100 \mu \mathrm{m}$. (I-L) MTS assay. One day after the final transduction, untransduced cells were removed using puromycin selection for $24 \mathrm{~h}$, after which 5000 cells per sample were plated onto 96-well plates. Cell proliferation was determined by use of MTS reagents $48 \mathrm{~h}$ after plating. The relative number of cells in cultures transduced with active versus negative control sgRNAs was determined by normalizing the optical density at 490 nm of each MTS reaction to the average optical density of the negative control reactions. 
$24 \mathrm{~h}$ after plating (72 $\mathrm{h}$ after lentiviral delivery of Cas9 and guide RNA) (Supplemental Fig. S11A-C), suggesting that mutant KRAS disruption causes $K R A S$ mutant cells to die. The expression of Cas9 and sgRNAs in HT29 cells did not alter the number of colonies formed on culture dishes or in soft agar (Fig. 3D, $\mathrm{H})$, the number of cells measured by MTS reagents (Fig. 3L), or cell index (Supplemental Fig. S11D), suggesting that targeting mutant KRAS with Cas9 and sgRNA does not affect proliferation or survival of this wild-type KRAS-containing cell line. Furthermore, the finding that 35A9P17 results in indel generation at significantly high frequency at wild-type KRAS in HT29 cells (Fig. $2 F)$ indicates that these cells are not dependent on KRAS, which is in line with a previous report (Baumer et al. 2015).

Targeting mutant KRAS with CRISPRCas9 blocks tumor growth in vivo

We next evaluated whether targeting mutant KRAS can suppress tumor growth in vivo. To express the 35T9P17 sgRNA in vivo after tumor formation, Cas9-expressing SW403 cells were first generated using lentiviral vector transduction. The cells were subsequently transduced with another lentiviral vector that expresses the 35T9P17 sgRNA in a doxycycline-inducible manner (Supplemental Fig. S4B; Aubrey et al. 2015). Subcutaneous transplantation of these double-transduced cancer cells into athymic nude mice led to tumor formation over $14 \mathrm{~d}$. Then, doxycycline was administered to the mice to induce 35T9P17 sgRNA expression in the tumor cells. This guide RNA expression dramatically inhibited tumor growth (Fig. 4A,B); tumor weights measured at day 26 (12 d after the start of doxycycline treatment) in the doxycycline group were 7.2-fold lower than those in the control group (Fig. 4E,G). Similarly, tumor weights were 10-fold reduced by doxycycline-induced expression of sgRNA in a different cancer cell line, SW480, which is homozygous for c.35G $>$ T (Supplemental Fig. S12). These data suggest that targeting mutant KRAS with CRISPR-Cas9 can control tumor growth in vivo.

When similar experiments were conducted using HT29 cells, which carry only wild-type KRAS, tumor growth and final tumor sizes were not altered by doxycycline-induced induction of guide RNA expression (Fig. 4C,D,F,H), indicating that expression of Cas9 and mutant KRAS-targeting guide RNA does not affect the tumorigenesis, proliferation, or survival of cells that do not contain mutant KRAS. Taken together, these data indicate that tumor con-

$\mathbf{E}$
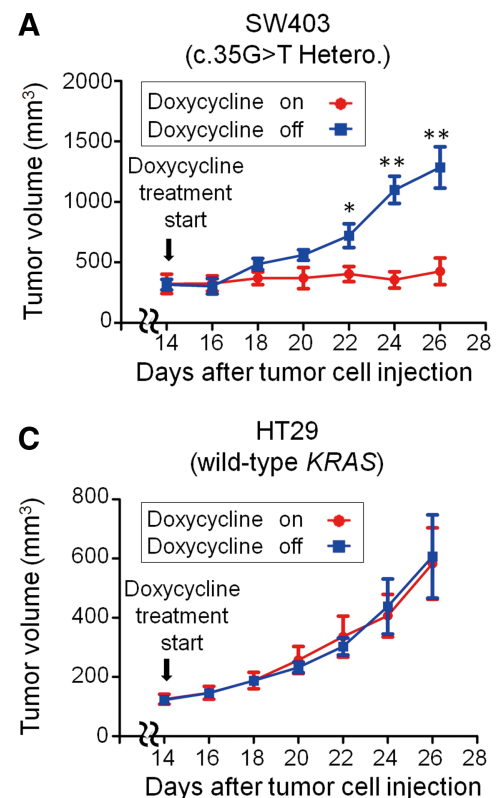

SW403 (c.35G>T Hetero.)

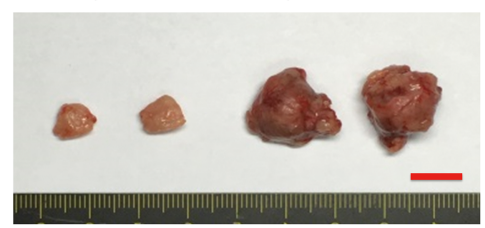

Doxycycline +
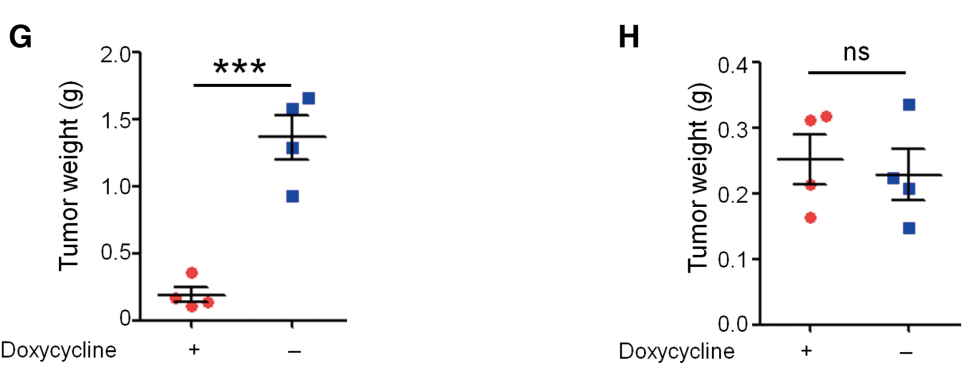

Figure 4. Targeting mutant KRAS with CRISPR-Cas9 blocks tumor growth in vivo. Cancer cells were transduced with a lentiviral vector expressing the 35T9P17 sgRNA in a doxycycline-inducible manner and a lentiviral vector that constitutively expresses Cas9. Transduced cells were subcutaneously injected into immunodeficient mice to allow tumor formation over $14 \mathrm{~d}$, after which the mice were treated with doxycycline to induce expression of 35T9P17. Tumor growth was monitored for $12 \mathrm{~d}$. (Hetero.) Heterozygous, (Homo.) homozygous. $(A, C)$ Tumor growth curves. Red and blue lines represent doxycycline-treated and untreated (control) groups, respectively. $(B, D)$ Representative photographs of the mice after $12 \mathrm{~d}$ of doxycycline treatment ( $26 \mathrm{~d}$ after tumor cell injection). (E-H) Representative photographs $(E, F)$ and weights $(G, H)$ of tumors removed from euthanized mice after $12 \mathrm{~d}$ of doxycycline treatment. Error bars represent SEM. $\left(^{*}\right) P<0.05,\left({ }^{* *}\right) P<0.01,\left({ }^{* * *}\right) P<0.001$. with the relevant KRAS mutations, which is in line with in vitro data shown above.

Intra-tumoral delivery of Cas9 and sgRNA targeting mutant KRAS suppresses tumor growth in vivo

We finally evaluated whether such Cas9 and sgRNA delivery can control human cancer growth in athymic mice. SW403

\section{Genome Research}

www.genome.org 
cancer cells that contained the KRAS c.35G $>\mathrm{T}$ mutation were subcutaneously injected into athymic mice, after which tumors were allowed to form over $14 \mathrm{~d}$. To deliver Cas9 and sgRNA into the cancer cells, we first used lentiviral vectors. Intra-tumoral injection of lentivirus expressing Cas9 and 35T9P17 sgRNA inhibited the tumor growth, which was in contrast to the robust tumor growth in the control group injected with lentivirus expressing only Cas9 (Fig. 5A-C; Supplemental Fig. S13A). Immunohistochemical staining using an antibody against puromycin acetyltransferase (PuroR), a protein that is expressed by the lentiviral vector for 35T9P17 sgRNA and Cas9 expression (Supplemental Fig. S4), showed that an average of $66 \%$ of tumor cells were positive for PuroR, indicating that Cas9 and 35T9P17 sgRNA were delivered to the majority, but not all, of the tumor cells (Supplemental Fig. S14A-E). The average indel frequency at the target site at $6 \mathrm{~d}$ after the first lentiviral injection was $27.3 \%$ in tumor cells (Supplemental Fig. S14F). Considering that SW403 cells also contain a wild-type $K R A S$ allele that is barely targeted by the $35 \mathrm{~T} 9 \mathrm{P} 17$ sgRNA, $\sim 55 \%(=27.3 \% \times 2)$ of tumor cells would be expected to contain indels at the target sites. We also used adeno-associated viral (AAV) vectors to deliver Cas9 and sgRNA into tumor cells, which likewise resulted in significant control of tumor growth, albeit to a lesser extent than caused by lentiviral delivery (Supplemental Fig. S15). Lentiviral delivery of Cas9 and sgRNA into tumors generated using cancer cells without mutant KRAS did not affect tumor growth, which was comparable to the growth of those injected with lentivirus expressing only Cas9 (Fig. 5D-F; Supplemental Fig. S13B), corroborating the specificity of 35T9P17 sgRNA for cancer cells containing the $\mathrm{c} .35 \mathrm{G}>\mathrm{T}$ mutation.

\section{Possibility of secondary oncogenic mutations in cells treated with Cas9 and sgRNA}

Given that targeting KRAS using Cas9 and sgRNA can induce various secondary mutations at or near the target sites, we cannot rule out the possibility that some of these secondary mutations might be oncogenic. To assess this point, we evaluated the genomic DNA of human cells treated with Cas9 and sgRNA using deep sequencing. Reported KRAS oncogenic mutations include G12D, G12V, G12C, G13D, G12R, G12A, and G12S (Cox and Der 2010). We found that these secondary oncogenic mutations were barely observed in either KRAS-mutant cancer cells (SW403) or cells containing wild-type KRAS (HT29) either in vitro or in vivo after lentiviral delivery of Cas9 and 35T9P17 sgRNA: The frequencies of mutations were similar to those of mutations in cells transduced with lentivirus that expressed only Cas9 (negative control) (Supplemental Fig. S16A-C). We also observed that frequencies of uncharacterized secondary nonsynonymous point mutations that are not included in the reported oncogenic mutation list described above were similar to those of negative controls, cells transduced with the lentiviral vector expressing only Cas9 (Supplemental Fig. S16D-F). These results suggest that secondary oncogenic point mutations are barely generated by Cas9 and sgRNA-directed targeting of KRAS. However, we cannot rule out the possibilities that targeting by Cas 9 and sgRNA could induce

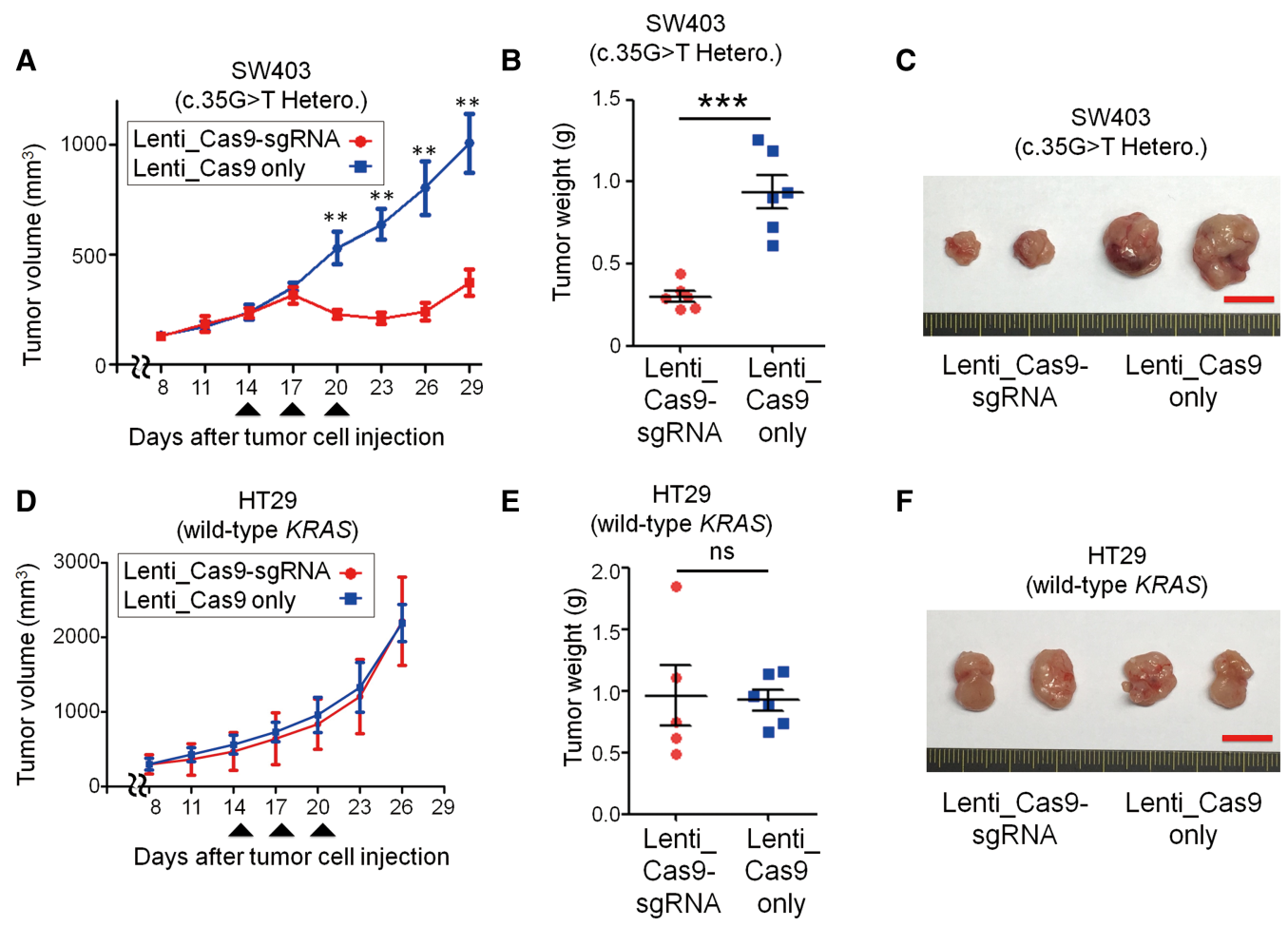

Figure 5. Intra-tumoral delivery of Cas 9 and sgRNA targeting mutant KRAS suppresses tumor growth in vivo in immunodeficient mice. Cancer cells containing mutant $K R A S(A-C)$ or wild-type $K R A S(D-F)$ were subcutaneously injected into immunodeficient mice, after which tumors were allowed to form over $14 \mathrm{~d}$. Lentivirus expressing both Cas9 and sgRNA (35T9P17) (Lenti_Cas9-sgRNA) or control lentivirus expressing only Cas9 (Lenti_Cas9) were injected into the tumors three times every $3 \mathrm{~d}$. $(A, D)$ Tumor growth curves. Black arrowheads indicate the times when the lentivirus was injected. The number of tested tumors $(n)=6$. $(B, C, E, F)$ Representative photographs $(B, E)$ and weights $(C, F)$ of tumors removed from euthanized mice $15 \mathrm{~d}(B, C)$ or $12 \mathrm{~d}(E, F)$ after the first lentiviral injection. Error bars represent SEM. $\left({ }^{* *}\right) P<0.01,\left({ }^{* *}\right) P<0.001$; (ns) not significant. 
secondary oncogenic point mutations at a frequency below the detection limit (negative control) and that some in-frame indels could potentially be oncogenic. Given that these secondary point mutations and indels would be resistant to targeting by the sgRNA that was initially used, we should consider the possibility that clones resistant to targeting by Cas 9 and the initially used sgRNA could be generated when we use Cas9 and sgRNA for tumor mass reduction.

\section{Discussion}

Programmable nucleases enable the targeting of specific genomic sequences, and we now show that cells containing specific cancer-causing sequences can be selectively controlled both in vitro and in vivo using CRISPR-Cas. In addition to mutant KRAS, other genes bearing cancer-associated mutations can be disrupted using CRISPR-Cas (Chen et al. 2017; Gebler et al. 2017; Koo et al. 2017); among these three studies, only two showed in vivo targeting of cancer-associated mutations, which included mutant epidermal growth factor receptor (EGFR) (Koo et al. 2017) and genomic rearrangements (TMEM135-CCDC67 and MAN2A1-FER fusions) (Chen et al. 2017), that led to control of tumor growth in mouse xenograft models. Compared to these two studies, our study is unprecedented in that we target $K R A S$, the most frequently mutated oncogene that cannot be targeted using any other clinically available anti-cancer agents such as small molecules or antibodies; mutant EGFR can be targeted using clinically available antibodies such as gefitinib and erlotinib, and TMEM135-CCDC67 and MAN2A1FER fusions are much less frequent than KRAS mutations. Furthermore, the two studies required a combination of two adenoviral vectors that deliver different cargos (Chen et al. 2017; Koo et al. 2017), whereas our study used only one lentiviral vector, simplifying the delivery component and expanding the list of possible delivery methods. In addition, our approach does not require any additional components such as ganciclovir (Chen et al. 2017) to control tumor growth in vivo. This control of mammalian cells containing specific sequences has parallels with sequence-specific control exhibited in microbials (Bikard et al. 2014; Citorik et al. 2014). Sequence-specific antimicrobial activity can distinguish a single-base change between two bacterial strains (Citorik et al. 2014), which is in line with our study showing that the selected guide RNAs can distinguish between mutant and wild-type $K R A S$, which differ by one base, in human cells. Our approach differentiates cancer cells from normal cells based on the presence of the oncogenic mutations themselves, targeting "the root" of cancer rather than subsequent changes that originate from the root.

So far, viral and nonviral approaches have been used to deliver transgenes into cancer cells (Yin et al. 2014; Naldini 2015). Due to possible adverse effects of delivery into normal cells, scientists have made substantial efforts to deliver transgenes specifically into cancer cells, which often restricts the choice of effective delivery methods. Our approach is compatible with nonselective methods that can deliver transgenes into both normal and cancer cells because cells that lack target sequences should not experience significant adverse effects in the presence of Cas9 and guide RNA, as we have shown in this study. However, we also cannot rule out the possibility that Cas9 and sgRNA delivered to normal cells could elicit gain-of-function mutations in the KRAS gene at an extremely low frequency that is below the detection limit of the current study.

Furthermore, transient delivery of Cas9 and guide RNA is sufficient for permanent target gene disruption, which is in contrast with small interfering RNAs, which need continuous delivery for target RNA inhibition (Zorde Khvalevsky et al. 2013). In summary, our approach is compatible with a wide variety of delivery methods, including those that are transient or nonselective.

Our study provides a proof-of-concept that CRISPR-Cas9 can be harnessed to target driver mutations in cancer without significant alterations of normal genes to inhibit cancer growth both in vitro and in vivo. Because the general mechanism of selective target sequence recognition and subsequent gene disruption that we utilized here is shared by other programmable nucleases such as zinc finger nucleases, transcription activator-like effector nucleases, and other types of CRISPR-Cas such as Cpf1 (Zetsche et al. 2015), tumor control may also be achieved using these other systems. Given that this tumor-controlling mechanism of CRISPRCas differs from those of previously reported cancer therapies, including conventional chemotherapy, radiotherapy, specific inhibitor or antibody therapy, and immunotherapy, combining CRISPR-Cas with these other therapies could lead to additive or synergistic effects. However, we also should note that the editing efficiency of CRISPR on the oncogene is not $100 \%$ and that the delivery efficiency of Cas9 and sgRNA to cancer cells is also not $100 \%$, both of which would prevent this strategy from causing complete tumor remission. Furthermore, we cannot rule out the possibilities that Cas9-induced mutagenesis at the wild-type allele could result in, albeit at extremely low efficiency, the generation of oncogenic alleles and that the mutagenesis at the oncogenic mutant allele could lead to the generation of other oncogenic mutant alleles, which are resistant to targeting by the initially designed sgRNA. Taken together, these issues indicate that CRISPR-Cas would not be able to induce tumor remission in human patients as a monotherapy, although it could be considered for reducing the tumor volume before subsequent therapies such as surgery. Furthermore, our successful application of CRISPR-Cas for targeting cancer driver mutations in vitro and in vivo can be utilized for investigating the functional and regulatory pathways of oncogenic drivers.

\section{Methods}

\section{Cell lines and culture conditions}

HEK293T cells (ATCC, CRL-11268) were purchased from the American Type Culture Collection (ATCC). HT29, SW403, SW480, SW620, LS513, and LoVo cells were obtained from the Korean Cell Line Bank (KCLB). Details on the cell culture media are in the Supplemental Methods.

\section{Plasmids encoding Cas9 and sgRNA}

A plasmid encoding Cas9, CMV promoter-Cas9-2A-mRFP-2A-Puro (hereafter, Cas9-Puro), was purchased from ToolGen. Cas9-Puro was prepared by inserting 2A-mRFP-2A-Puro into pSpCas9(BB)2A-Puro V2.0 (Addgene, \#62988). For sgRNA expression, the hU6-sgRNA plasmid (ToolGen) was used. Oligonucleotides containing each target sequence were synthesized (Bioneer) and then annealed in vitro in a thermocycler. Annealed oligonucleotides were ligated into the hU6-sgRNA plasmid digested with BsaI.

\section{Reporter assay}

Reporters containing target sequences of interest were prepared as previously described (Ramakrishna et al. 2014). Briefly, oligonucleotides containing target sequences (Fig. 1; Supplemental Fig. S3) were synthesized (Bioneer) and annealed in vitro in a

\section{Genome Research}

www.genome.org 
thermocycler. The annealed oligonucleotides were ligated into the pRGS vector (Ramakrishna et al. 2014) predigested with EcoR1 and BamH1. HEK293T cells were transfected with mixtures of the Cas9-puro, U6-sgRNA, and reporter plasmids at a weight ratio of 1:1:1 using Neon electroporation (Invitrogen). Three days after transfection, adherent cells were trypsinized and resuspended in $\mathrm{Ca}^{2+}$ - and $\mathrm{Mg}^{2+}$-free PBS (Gibco-BRL) supplemented with $2 \%$ FBS. Single-cell suspensions were analyzed with FACSAria II (BD Biosciences). We then calculated the percentage of $\mathrm{mRFP}^{+} \mathrm{eGFP}^{+}$ cells among the total population of $\mathrm{mRFP}^{+}$cells, which represented the activity of the guide RNA on the target sequence.

\section{Focus-forming assay}

Cells double-transduced with lentivirus expressing Cas9 (Supplemental Fig. S4C) and lentivirus expressing sgRNA (Supplemental Fig. S4A) as described above were seeded in six-well plates at a density of 1000 cells/well. After incubation for $10 \mathrm{~d}$, the resulting colonies were rinsed with PBS, stained with $2 \%$ crystal violet, photographed, and counted using ImageJ (Schneider et al. 2012).

\section{Anchorage independence culture assay}

The culture platform consisted of two soft agar (Dongin-Genomic) layers (top, 0.4\% agar; bottom, 0.6\% agar) in DMEM supplemented with $10 \%$ FBS in 60 -mm dishes. Cells $\left(10^{5}\right.$ per well) doubletransduced with lentivirus expressing Cas9 and lentivirus expressing sgRNA as described above were encapsulated in the top layer. After $3 \mathrm{wk}$, colonies were stained with crystal violet for $16 \mathrm{~h}$.

\section{MTS cell proliferation assay}

Cells double-transduced with lentivirus expressing Cas9 and lentivirus expressing sgRNA were plated in 96-well culture plates at $1 \times$ $10^{4}$ cells per well. MTS assays were performed $12,24,48$, and $72 \mathrm{~h}$ after cell plating. MTS (3-[4,5-dimethylthiazol-2-yl]-5-[3-carboxymethoxyphenyl]-2-[4-sulfophenyl]-2H-tetrazolium; CellTiter 96 Aqueous One Solution Cell Proliferation Assay, Promega) was added to each well at a proportion of $1 / 10$, after which plates were incubated for $4 \mathrm{~h}$ at $37^{\circ} \mathrm{C}$. The medium was then removed and 180 $\mu \mathrm{L}$ DMSO was added to each well. The absorbance at $490 \mathrm{~nm}$ was measured by a microplate reader (multiscan MK3; Thermo Fisher Scientific). The mean cell proliferation was calculated from the absorbance units.

\section{Real-time cell analysis (RTCA)}

Cells were double-transduced with lentivirus expressing sgRNA and lentivirus expressing SpCas9, each at about 10-20 MOI. Cells were added to the sensor wells of an E-plate 16 (Roche) at a density of 5000 cells/well and incubated for $24 \mathrm{~h}$ before analysis. Fifty microliters of DMEM was added to each well for the background measurement. The E-plate was incubated in the presence of $5 \% \mathrm{CO}_{2}$ at $37^{\circ} \mathrm{C}$ and monitored using the RTCA SP system (Roche) for $48 \mathrm{~h}$ with time intervals of at least $15 \mathrm{~min}$. Analysis was performed using RTCA software version 1.2.1 (Roche).

\section{Analysis of indel frequency}

Genomic DNA was isolated from cells after $72 \mathrm{~h}$ of lentiviral transduction using a Wizard Genomic DNA Purification kit (Promega). KRAS target sequences were PCR-amplified by Pfu DNA Polymerase (SolGent) for indel frequency analysis. We used 13 $\mu \mathrm{g}$ of genomic DNA per sample as a template for the first PCR. PCR products were purified with a MEGAquick-spin Total Fragment DNA Purification kit (Intron). For the second PCR, 20 ng of purified PCR products from the first amplification were an- nealed with both Illumina adaptor and barcode sequences. The resulting products were isolated, purified, mixed, and analyzed using MiSeq or HiSeq (Illumina). Deep-sequencing data were sorted and analyzed by previously described Python scripts (Kim et al. 2017a). Data sorting was conducted based on 19-base barcode sequences. Insertions or deletions located around the expected cleavage site (i.e., an 8-bp region centered on the cleavage site) were considered to be CRISPR-Cas9-induced mutations. Single-base substitutions were exempted from the analysis. For secondary mutation analysis, sequencing reads were mapped to the reference KRAS sequence (NCBI) using the Burrows-Wheeler alignment algorithm (Li and Durbin 2009). Mutations were then measured by an in-house Python program (see Data access) and SAMtools (Li et al. 2009).

\section{Intra-tumoral injection of lentivirus or AAV expressing Cas9 and sgRNA}

Two million cancer cells were subcutaneously injected into the flanks of 5-wk-old athymic male BALB/c nude mice (six mice per group) and were allowed to form tumors over $2 \mathrm{wk}$. Each tumor was directly injected with $1 \times 10^{8} \mathrm{TU}$ lentivirus in $50 \mu \mathrm{L}$ PBS or with $1 \times 10^{12} \mathrm{gc} / \mathrm{ml} \mathrm{AAV}$ in $50 \mu \mathrm{L}$ PBS with insulin syringes (BD Biosciences, 31 gauge) three times with intervals of $3 \mathrm{~d}$ between injections. Tumor size was evaluated every $3 \mathrm{~d}$ using a caliper. Mice were sacrificed $5 \mathrm{wk}$ after the tumor cell injection, and the tumors were surgically isolated and weighed.

\section{Immunohistochemistry}

Mice were sacrificed by carbon dioxide asphyxiation. Tumors were fixed with $4 \%$ paraformaldehyde, embedded in paraffin, sectioned at $5 \mu \mathrm{m}$, and stained with hematoxylin and eosin (H\&E) for histological examination. Tumor sections were de-waxed, rehydrated, and stained using standard immunohistochemistry protocols. The following antibody was used: anti-puromycin acetyltransferase (PuroR) (1:500) (Kim et al. 2017b). The numbers of PuroR-positive and -negative tumor cells were quantified from $>3$ regions per tumor. A total of at least 1000 tumor cells/tumor were counted.

\section{Statistical analysis}

All data were expressed as means \pm SEM, and statistical analysis was conducted using IBM SPSS statistics (version 21). Student's $t$-test was used for the statistical analysis for continuous variables between two groups and ANOVA followed by multiple comparison with Bonferroni's method for variables among more than two groups. A $P$ value $<0.05$ was considered statistically significant.

\section{Data access}

The deep sequencing data from this study have been submitted to the NCBI Sequence Read Archive (SRA; http://www.ncbi.nlm.nih. gov/sra/) under accession number SRP124382. A custom Python code for mutation analysis is available at GitHub (https://github. com/CRISPRJWCHOI/KRAS_CRISPR_CAS9) and as Supplemental Code.

\section{Acknowledgments}

The authors thank D.-S. Jang, MFA (Medical Illustrator, Medical Research Support Section, Yonsei University College of Medicine, Seoul, Korea), for his help with the illustrations. This work was supported in part by the National Research Foundation of Korea (2017R1A2B3004198, 2017M3A9B4062403, 2014M3A9B6073 507, and 2013M3A9B4076544), Institute for Basic Science (IBS; IBS-R026-D1), and the Korean Health Technology R\&D Project, 
Ministry of Health and Welfare, Republic of Korea (HI17C0676 [Medistar programme], HI14C1324).

\section{References}

Athuluri-Divakar SK, Vasquez-Del Carpio R, Dutta K, Baker SJ, Cosenza SC, Basu I, Gupta YK, Reddy MV, Ueno L, Hart JR, et al. 2016. A small molecule RAS-mimetic disrupts RAS association with effector proteins to block signaling. Cell 165: 643-655.

Aubrey BJ, Kelly GL, Kueh AJ, Brennan MS, O'Connor L, Milla L, Wilcox S, Tai L, Strasser A, Herold MJ. 2015. An inducible lentiviral guide RNA platform enables the identification of tumor-essential genes and tumor-promoting mutations in vivo. Cell Rep 10: 1422-1432.

Baumer S, Baumer N, Appel N, Terheyden L, Fremerey J, Schelhaas S, Wardelmann E, Buchholz F, Berdel WE, Muller-Tidow C. 2015. Antibody-mediated delivery of anti-KRAS-siRNA in vivo overcomes therapy resistance in colon cancer. Clin Cancer Res 21: 1383-1394.

Bikard D, Euler CW, Jiang W, Nussenzweig PM, Goldberg GW, Duportet X, Fischetti VA, Marraffini LA. 2014. Exploiting CRISPR-Cas nucleases to produce sequence-specific antimicrobials. Nat Biotechnol 32: 11461150 .

Brummelkamp TR, Bernards R, Agami R. 2002. Stable suppression of tumorigenicity by virus-mediated RNA interference. Cancer Cell 2: 243-247.

Chen ZH, Yu YP, Zuo ZH, Nelson JB, Michalopoulos GK, Monga S, Liu S, Tseng G, Luo JH. 2017. Targeting genomic rearrangements in tumor cells through Cas9-mediated insertion of a suicide gene. Nat Biotechnol 35: $543-550$.

Citorik RJ, Mimee M, Lu TK. 2014. Sequence-specific antimicrobials using efficiently delivered RNA-guided nucleases. Nat Biotechnol 32: 1141-1145.

Cong L, Ran FA, Cox D, Lin S, Barretto R, Habib N, Hsu PD, Wu X, Jiang W, Marraffini LA, et al. 2013. Multiplex genome engineering using CRISPR/ Cas systems. Science 339: 819-823.

Cox AD, Der CJ. 2010. Ras history: The saga continues. Small GTPases 1: $2-27$.

Cox AD, Fesik SW, Kimmelman AC, Luo J, Der CJ. 2014. Drugging the undruggable RAS: mission possible? Nat Rev Drug Discov 13: 828-851.

Gebler C, Lohoff T, Paszkowski-Rogacz M, Mircetic J, Chakraborty D, Camgoz A, Hamann MV, Theis M, Thiede C, Buchholz F. 2017. Inactivation of cancer mutations utilizing CRISPR/Cas9. J Natl Cancer Inst 109: djw183.

Hunter JC, Manandhar A, Carrasco MA, Gurbani D, Gondi S, Westover KD. 2015. Biochemical and structural analysis of common cancer-associated KRAS mutations. Mol Cancer Res 13: 1325-1335.

Jinek M, Chylinski K, Fonfara I, Hauer M, Doudna JA, Charpentier E. 2012. A programmable dual-RNA-guided DNA endonuclease in adaptive bacterial immunity. Science 337: 816-821.

Kim H, Kim JS. 2014. A guide to genome engineering with programmable nucleases. Nat Rev Genet 15: 321-334.

Kim H, Um E, Cho SR, Jung C, Kim H, Kim JS. 2011. Surrogate reporters for enrichment of cells with nuclease-induced mutations. Nat Methods 8 : 941-943.
Kim YH, Kim HO, Baek EJ, Kurita R, Cha HJ, Nakamura Y, Kim H. 2015. Rh D blood group conversion using transcription activator-like effector nucleases. Nat Commun 6: 7451.

Kim HK, Song M, Lee J, Menon AV, Jung S, Kang YM, Choi JW, Woo E, Koh HC, Nam JW, et al. 2017a. In vivo high-throughput profiling of CRISPRCpf1 activity. Nat Methods 14: 153-159.

Kim HS, Cheon JH, Jung ES, Park J, Aum S, Park SJ, Eun S, Lee J, Ruther U, Yeo GSH, et al. 2017b. A coding variant in FTO confers susceptibility to thiopurine-induced leukopenia in East Asian patients with IBD. Gut 66: $1926-1935$

Koo T, Yoon AR, Cho HY, Bae S, Yun CO, Kim JS. 2017. Selective disruption of an oncogenic mutant allele by CRISPR/Cas9 induces efficient tumor regression. Nucleic Acids Res 45: 7897-7908.

Li H, Durbin R. 2009. Fast and accurate short read alignment with BurrowsWheeler transform. Bioinformatics 25: 1754-1760.

Li H, Handsaker B, Wysoker A, Fennell T, Ruan J, Homer N, Marth G, Abecasis G, Durbin R; Genome Project Data Processing Subgroup. 2009. The Sequence Alignment/Map format and SAMtools Bioinformatics 25: 2078-2079.

Mali P, Yang L, Esvelt KM, Aach J, Guell M, DiCarlo JE, Norville JE, Church GM. 2013. RNA-guided human genome engineering via Cas9. Science 339: $823-826$.

Naldini L. 2015. Gene therapy returns to centre stage. Nature 526: 351-360.

Ostrem JM, Peters U, Sos ML, Wells JA, Shokat KM. 2013. K-Ras(G12C) inhibitors allosterically control GTP affinity and effector interactions. Nature 503: 548-551.

Patricelli MP, Janes MR, Li LS, Hansen R, Peters U, Kessler LV, Chen Y, Kucharski JM, Feng J, Ely T, et al. 2016. Selective inhibition of oncogenic KRAS output with small molecules targeting the inactive state. Cancer Discov 6: 316-329.

Ramakrishna S, Cho SW, Kim S, Song M, Gopalappa R, Kim JS, Kim H. 2014 Surrogate reporter-based enrichment of cells containing RNA-guided Cas9 nuclease-induced mutations. Nat Commun 5: 3378.

Schneider CA, Rasband WS, Eliceiri KW. 2012. NIH Image to ImageJ: 25 years of image analysis. Nat Methods 9: 671-675.

Shi J, Wang E, Milazzo JP, Wang Z, Kinney JB, Vakoc CR. 2015. Discovery of cancer drug targets by CRISPR-Cas9 screening of protein domains. Nat Biotechnol 33: 661-667.

Yin H, Kanasty RL, Eltoukhy AA, Vegas AJ, Dorkin JR, Anderson DG. 2014 Non-viral vectors for gene-based therapy. Nat Rev Genet 15: 541-555.

Zetsche B, Gootenberg JS, Abudayyeh OO, Slaymaker IM, Makarova KS, Essletzbichler P, Volz SE, Joung J, van der Oost J, Regev A, et al. 2015. Cpf1 is a single RNA-guided endonuclease of a class 2 CRISPR-Cas system. Cell 163: 759-771.

Zorde Khvalevsky E, Gabai R, Rachmut IH, Horwitz E, Brunschwig Z, Orbach A, Shemi A, Golan T, Domb AJ, Yavin E, et al. 2013. Mutant KRAS is a druggable target for pancreatic cancer. Proc Natl Acad Sci 110: 20723-20728.

Received April 11, 2017; accepted in revised form January 8, 2018.

\section{Genome Research}

www.genome.org 


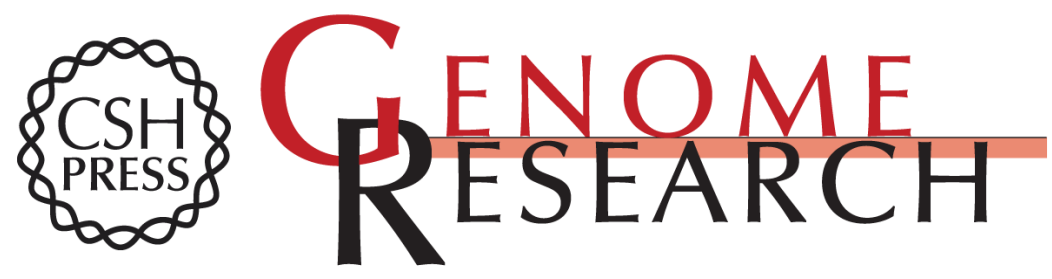

\section{Targeting mutant KRAS with CRISPR-Cas9 controls tumor growth}

Wonjoo Kim, Sangeun Lee, Han Sang Kim, et al.

Genome Res. 2018 28: 374-382 originally published online January 11, 2018

Access the most recent version at doi:10.1101/gr.223891.117

\section{Supplemental http://genome.cshlp.org/content/suppl/2018/02/09/gr.223891.117.DC1 \\ Material}

References This article cites 31 articles, 8 of which can be accessed free at:

http://genome.cshlp.org/content/28/3/374.full.html\#ref-list-1

Creative This article is distributed exclusively by Cold Spring Harbor Laboratory Press for the Commons

License first six months after the full-issue publication date (see

http://genome.cshlp.org/site/misc/terms.xhtml). After six months, it is available under a Creative Commons License (Attribution-NonCommercial 4.0 International), as described at http://creativecommons.org/licenses/by-nc/4.0/.

Email Alerting Receive free email alerts when new articles cite this article - sign up in the box at the Service top right corner of the article or click here.

\section{Affordable, Accurate Sequencing.}

To subscribe to Genome Research go to:

https://genome.cshlp.org/subscriptions 